\section{Características biológicas e evitabilidade de óbitos perinatais em uma localidade na cidade do Rio de Janeiro, 1999 a 2003}

\section{Biological features and avoidability of perinatal deaths in the city of Rio de Janeiro locality from 1999 to 2003}

Sandra Costa Fonseca 1

Evandro da Silva Freire Coutinho 2

\section{Abstract}

Objectives: to analyze perinatal deaths in a public maternity hospital, located in the city of Rio de Janeiro, Brazil, according to biological characteristics, basic causes, avoidability and birth weightspecific mortality rates.

Methods: the coorte study was performed from 1999 to 2003 in the maternity Leila Diniz, Hospital Municipal Raphael de Paula Souza, assisting 50\% of the births in the geographic/administrative area 4 (Jacarepaguá and Barra da Tijuca). Perinatal deaths in cases where birth weight was equal or superior to $500 \mathrm{~g}$ were included. A standardized tool-Perinatal Investigation Report - and medical records were used to collect data. Death causes were investigated and the Wigglesworth's Classification was applied to assess deaths' avoidability.

Results: there were 512 perinatal deaths (377 stillborns and 135 early neonatal deaths). Neonatal deaths tended to occur in the first three days of life. Twenty-four percent of the perinatal deaths occurred in newborns smaller than expected in relation to gestational age. The principal causes of death were maternal morbidities (hypertension and syphilis) and placental complications. According to Wigglesworth's Classification, $50 \%$ of the deaths were avoidable.

Conclusions: the number of avoidable deaths was high, mainly stillbirths, and referred to conditions related to prenatal care

Key words Perinatal mortality, Public health, Prenatal care
1 Departamento de Epidemiologia e Bioestatística. Instituto de Saúde da Comunidade. Universidade Federal Fluminense. Rua Marquês de Paraná, 303. 3. andar. Prédio Anexo ao Hospital Universitário Antonio Pedro. Niterói, RJ, Brasil. CEP: 24.030-210. E-mail: sandracfonseca@yahoo.com.br

2 Departamento de Epidemiologia e Métodos Quantitativos em Saúde. Escola Nacional de Saúde Pública Sérgio Arouca. Fundação Oswaldo Cruz. Rio de Janeiro, RJ, Brasil.

\section{Resumo}

Objetivos: analisar os óbitos perinatais de uma maternidade pública do municipio do Rio de Janeiro, Brasil, quanto à características biológicas, critérios de evitabilidade, causas básicas e mortalidade por faixas de peso.

Métodos: o estudo de coorte foi realizado de 1999 a 2003, na Maternidade Leila Diniz do Hospital Municipal Raphael de Paula Souza, que é responsável por $50 \%$ dos partos de uma área programática 4 (Jacarepaguá e Barra da Tijuca). Foram incluídos óbitos perinatais com peso igual ou superior a $500 \mathrm{~g}$. O instrumento principal de coleta de dados foi a Ficha de Investigação de Óbito Perinatal. Foi aplicada a Classificação de Wigglesworth para avaliar evitabilidade.

Resultados: houve 512 óbitos perinatais (377 fetais e 135 neonatais precoces). Os óbitos neonatais se concentraram nos primeiros três dias de vida. Vinte e quatro por cento dos óbitos cursaram com retardo de crescimento intra-uterino. As principais causas foram afecções maternas (hipertensão e sífilis) e complicações de membrana, placenta e cordão. Foram considerados evitáveis cerca de $50 \%$ dos óbitos.

Conclusões: o percentual de óbitos evitáveis foi elevado, mais concentrado nos óbitos fetais ocorridos antes do parto, sugerindo inadequação do pré-natal.

Palavras-chave Mortalidade perinatal, Causa básica de morte, Cuidado pré-natal 


\section{Introdução}

A mortalidade perinatal, apesar do decréscimo na última década, ainda é elevada no Brasil, com diferenças importantes nos níveis nacional e regional. Uma revisão dos estudos mais recentes ${ }^{1}$ mostrou que a natimortalidade variou de $9 \%$ em Belo Horizonte a 17,9\% em Fortaleza, enquanto a neomortalidade precoce variou de 7,4\% em Caxias do Sul a $15,3 \%$ em Fortaleza. Além das diferenças na magnitude, observou-se heterogeneidade em diversos aspectos metodológicos, prejudicando a comparabilidade dos estudos. Poucos trabalhos ${ }^{2-5}$ avaliaram a evitabilidade de óbito perinatal, sendo a Classificação de Wigglesworth, ${ }^{6}$ adaptada por Keeling et al., ${ }^{7}$ a mais citada.

O estudo da mortalidade perinatal em nível regional tem sido enfatizado, 8 e a aplicação de metodologias para avaliar causas básicas e evitabilidade é fundamental para se planejar adequadamente as ações na área perinatal. ${ }^{9}$

Na cidade do Rio de Janeiro, a mortalidade perinatal no ano de 2003 foi de $16,8 \%, 10$ sendo a fetal $9,2 \%$ e a neonatal precoce $7,6 \%$. Pela dimensão geográfica e diferença socioeconômica do município, observam-se variações das taxas de mortalidade perinatal entre as regiões administrativas (RA), de 9,3 a $23,2 \%$. 10

$\mathrm{Na}$ área programática 4 (AP4), que compreende as regiões de Jacarepaguá e Barra da Tijuca, com cerca de 700.000 habitantes, existe uma diferença relevante na mortalidade perinatal: $17,6 \%$ na RA de Jacarepaguá e 8,2\%o na RA da Barra da Tijuca. 10 A Maternidade Leila Diniz, pertencente ao Hospital Raphael de Paula Souza da Secretaria Municipal de Saúde, Rio de Janeiro, Brasil, era a única maternidade pública da área, responsável por cerca de 50 a $60 \%$ dos partos de usuárias do Sistema Único de Saúde (SUS) que residem na AP4, concentrando-se o atendimento aos bairros adstritos à maternidade, na região de Jacarepaguá. Sessenta por cento dos óbitos perinatais $(80 \%$ dos óbitos fetais e $30 \%$ dos óbitos neonatais precoces) da AP4 ocorriam nesta unidade e refletiam, de forma aproximada, a situação perinatal dessa população.

O objetivo deste estudo foi descrever e analisar os óbitos perinatais atendidos na Maternidade Leila Diniz, de 1999 a 2003. Os aspectos destacados foram: coeficientes de mortalidade por faixa de peso; características biológicas dos fetos e recém-nascidos (RN); classificação por evitabilidade e causas básicas.

\section{Métodos}

O estudo retrospectivo das coortes de nascimento de 1999 a 2003, incluiu as pacientes residentes na AP4, atendidas na Maternidade Leila Diniz, cujas gestações terminaram em óbito perinatal - óbito neonatal precoce (menos que sete dias de vida) ou óbito fetal com idade gestacional igual ou superior a 22 semanas. Foram incluídos os óbitos com peso igual ou superior a $500 \mathrm{~g}$.

O instrumento principal para coleta de dados "Ficha de Investigação de Óbito Fetal e Neonatal" continha variáveis socioeconômicas e demográficas, histórico obstétrico materno, dados da assistência pré-natal, obstétrica e neonatal e características biológicas do feto. $11 \mathrm{O}$ preenchimento foi realizado à medida que os óbitos ocorriam, pelos obstetras, neonatologistas e epidemiologistas do serviço. O armazenamento dos dados foi feito no programa EpiInfo 6.04. Para a realização do estudo, todas as fichas e os respectivos prontuários foram revistos a fim de complementar as informações ausentes, confirmar a causa básica de óbito e aplicar a classificação de evitabilidade.7

Em função da pequena variabilidade anual dos coeficientes de mortalidade por faixa de peso, optouse pela análise dos coeficientes globais no período. Para o cálculo dos coeficientes de mortalidade perinatal e fetal utilizou-se, no denominador, o total de nascimentos (nascidos vivos e natimortos) ocorridos na Maternidade Leila Diniz, entre as mães residentes na AP4. No cálculo do coeficiente de mortalidade neonatal, foram incluídos no denominador apenas os nascidos vivos. Os dados foram obtidos do Sistema de Informação de Nascidos Vivos (SINASC) e do Sistema de Informação de Mortalidade (SIM) do Rio de Janeiro, que foram gentilmente cedidos pela Coordenação de Epidemiologia da Secretaria Municipal de Saúde.

As características biológicas - peso, idade gestacional e crescimento intra-uterino foram coletadas das fichas, dos prontuários e livros de registro da maternidade. Os pesos dos óbitos fetais foram aferidos pela enfermagem e os pediatras pesaram todos os recém-nascidos, na sala de parto.

A informação sobre idade gestacional estava ausente ou discrepante em um percentual elevado de fichas. Os prontuários foram revistos e foi possível redefinir a idade gestacional (IG) de acordo com os seguintes critérios: a) priorização da data da última menstruação, caso concordante com ultra-sonografia realizada até a $24^{\mathrm{a}}$ semana; 12 b) utilização da ultrasonografia, quando não havia informação da data da última menstruação ou havia discordância maior que 
Classificação de Wigglesworth* modificada por Keeling et al.** e relação com falhas na assistência.

\begin{tabular}{|c|c|c|}
\hline & Tipo de óbito & Possivel falha na assistência \\
\hline Grupo 1 & Ocorrido antes do trabalho de parto (natimortos) & Ausência ou inadequação do pré-natal \\
\hline Grupo 2 & $\begin{array}{l}\text { Por malformações congênitas letais (natimortos } \\
\text { e neomortos) }\end{array}$ & $\begin{array}{l}\text { Falha no acompanhamento periconcepcional } \\
\text { e aconselhamento genético anterior }\end{array}$ \\
\hline Grupo 3 & Por prematuridade (neomortos) & $\begin{array}{l}\text { Falha no acompanhamento obstétrico e/ou } \\
\text { neonatal }\end{array}$ \\
\hline Grupo 4 & $\begin{array}{l}\text { Por asfixia perinatal (natimortos durante trabalho } \\
\text { de parto ou neomortos com asfixia grave) }\end{array}$ & $\begin{array}{l}\text { Falha na atenção obstétrica ou na } \\
\text { reanimação neonatal }\end{array}$ \\
\hline Grupo 5 & $\begin{array}{l}\text { Por causas específicas (natimortos e neomortos) } \\
\text { - infecç̃̃es, isoimunização }\end{array}$ & $\begin{array}{l}\text { Falhas no pré-natal, atendimento } \\
\text { obstétrico e/ou neonatal }\end{array}$ \\
\hline
\end{tabular}

Fonte: * Wigglesworth JS. Monitoring perinatal mortality. Lancet. 1980.6; ** Keeling JW et al. Classification of perinatal death. Arch Dis Child. 1989.7

duas semanas; c) emprego, tanto no feto quanto no recém-nascido, das medidas antropométricas do comprimento e perímetro cefálico, 13,14 na ausência da data da última menstruação e da ultra-sonografia; d) para os óbitos neonatais, além da antropometria, foi usada a estimativa clínica. 15,16 Dessa forma, foi criada uma variável, a idade gestacional estimada, que descreveu a melhor estimativa de idade gestacional, permitindo também a estimativa de crescimento intra-uterino.

Para avaliar o crescimento intra-uterino, foi utilizada a curva de Williams et al.,17 conforme recomendado pela Organização Mundial da Saúde. 18 Foi considerado indicativo de crescimento restrito o peso abaixo do percentil 10 para a idade gestacional correspondente.

Para avaliar a evitabilidade dos óbitos, foi utilizada a Classificação de Wigglesworth ${ }^{6}$ adaptada por Keeling et al.,7 que agrupa os óbitos perinatais em cinco categorias mutuamente excludentes, de base fisiopatológica e associadas a possíveis falhas na intervenção assistencial (Tabela 1). Os óbitos foram alocados nas categorias de acordo com algoritmo proposto por Keeling et al. ${ }^{7}$ Para avaliar as causas básicas, priorizou-se o evento inicial na cadeia causal, buscando morbidades que tivessem desencadeado o desfecho reprodutivo. As causas foram agrupadas de acordo com a $10^{\mathrm{a}}$ revisão da Classificação Estatística Internacional de Doenças. 19 Os dados foram armazenados e processados, utilizando o programa Epi-Info 6.04. Este estudo foi aprovado pelo Comitê de Ética da Escola Nacional de Saúde Pública e pela direção da Maternidade Leila Diniz.

\section{Resultados}

No período de janeiro de 1999 a dezembro de 2003, ocorreram 19.340 nascimentos na Maternidade Leila Diniz, de residentes da AP4. Houve 512 óbitos perinatais com peso $\geq 500 \mathrm{~g}$, sendo 377 fetais e 135 neonatais precoces. Estes últimos ocorreram predominantemente nos primeiros três dias de vida, sendo que metade no primeiro dia. As pacientes com óbito fetal já chegaram à maternidade com o feto morto em $84 \%$ dos casos. O restante ocorreu com a paciente já internada, antes $(8 \%)$ e durante o trabalho de parto $(8 \%)$.

O coeficiente de mortalidade perinatal oscilou em torno de $25 \%$ no período. A mortalidade fetal teve pouca variação, ficando próxima de $18 \% \mathrm{em}$ todos os anos. Já a mortalidade neonatal precoce manteve-se abaixo de $8 \%$, com o menor valor em $2001(5 \%)$.

As pacientes que tiveram óbito perinatal eram primíparas em $37 \%$ dos casos e multíparas de quatro ou mais gestações em $11 \%$. A idade média foi de 25 anos, com $22 \%$ de adolescentes e $14 \%$ com idade igual ou superior a 35 anos. O pré-natal foi ausente em $37 \%$ e apenas $16 \%$ realizaram seis ou mais consultas.

A Tabela 2 mostra os coeficientes de mortalidade para cada faixa de peso. Observam-se valores decrescentes de todos os coeficientes à medida que aumenta o peso ao nascer, tanto para o coeficiente de mortalidade perinatal como um todo quanto para os coeficientes de mortalidade fetal e neonatal precoce. A mediana de peso dos 512 óbitos perinatais foi $1420 \mathrm{~g}$, sendo que $77 \%$ dos óbitos tiveram peso menor que $2500 \mathrm{~g}$ e $52 \%$ abaixo de $1500 \mathrm{~g}$. 
Tabela 2

Número absoluto e coeficiente de mortalidade (por mil nascimentos) por faixa de peso (500 g) e faixas agregadas, óbitos perinatais, fetais e neonatais precoces. Maternidade Leila Diniz do Hospital Municipal Raphael de Paula Souza, Rio de Janeiro, 1999 a 2003.

\begin{tabular}{|c|c|c|c|c|c|c|c|}
\hline Peso ao nascer (g) & $\begin{array}{c}\text { Nascidos } \\
\text { vivos (AP4) }\end{array}$ & Óbitos fetais & $\begin{array}{l}\text { Coeficiente } \\
\text { mortalidade } \\
\text { fetal }\end{array}$ & $\begin{array}{c}\text { Óbitos } \\
\text { neonatais } \\
\text { precoces }\end{array}$ & $\begin{array}{c}\text { Coeficiente } \\
\text { mortalidade } \\
\text { neonatal precoce }\end{array}$ & $\begin{array}{c}\text { Óbitos } \\
\text { perinatais }\end{array}$ & $\begin{array}{c}\text { Coeficiente } \\
\text { mortalidade } \\
\text { perinatal }\end{array}$ \\
\hline \multicolumn{8}{|l|}{ Faixas etárias } \\
\hline $500-999$ & 148 & 113 & 432,9 & 61 & 412,1 & 174 & 666,6 \\
\hline $1000-1499$ & 212 & 74 & 258,7 & 20 & 94,3 & 94 & 328,6 \\
\hline 1500-1999 & 390 & 54 & 121,6 & 16 & 40,9 & 70 & 157,6 \\
\hline $2000-2499$ & 1244 & 46 & 35,6 & 10 & 8,0 & 56 & 45,0 \\
\hline$\geq 2500$ & 17.346 & 90 & 5,1 & 28 & 1,6 & 118 & 6,8 \\
\hline \multicolumn{8}{|c|}{ Faixas etárias agregadas } \\
\hline$<1500$ & 360 & 187 & 341,8 & 81 & 225,0 & 258 & 471,6 \\
\hline$<2500$ & 1994 & 287 & 125,8 & 107 & 53,7 & 384 & 168,3 \\
\hline Total & 19.340 & 377 & 19,0 & 135 & 7,0 & 512 & 25,9 \\
\hline
\end{tabular}

AP4=área programática 4; Faixas agregadas - cumulativas

Tabela 3

Número absoluto e percentual dos óbitos perinatais, fetais e neonatais por faixa de idade gestacional. Maternidade Leila Diniz do Hospital Municipal Raphael de Paula Souza, Rio de Janeiro, 1999 a 2003.

\begin{tabular}{|c|c|c|c|c|c|c|}
\hline \multirow{2}{*}{$\begin{array}{l}\text { Idade gestacional } \\
\text { em semanas } \\
\text { semanas }\end{array}$} & \multicolumn{2}{|c|}{$\begin{array}{c}\text { Óbitos } \\
\text { perinatais }\end{array}$} & \multicolumn{2}{|c|}{$\begin{array}{l}\text { Óbitos } \\
\text { fetais }\end{array}$} & \multicolumn{2}{|c|}{$\begin{array}{c}\text { Óbitos neonatais } \\
\text { precoces }\end{array}$} \\
\hline & $\mathrm{n}$ & $\%$ & $\mathrm{n}$ & $\%$ & $\mathrm{n}$ & $\%$ \\
\hline$<22$ & 6 & 1,0 & 6 & 1,5 & \multicolumn{2}{|c|}{0} \\
\hline 22 a 27 & 138 & 27,0 & 85 & 22,5 & 53 & 39,0 \\
\hline 28 a 31 & 103 & 20,0 & 75 & 20,0 & 28 & 21,0 \\
\hline 32 a 36 & 133 & 26,0 & 109 & 29,0 & 24 & 18,0 \\
\hline$\geq 37$ & 114 & 22,0 & 84 & 22,0 & 30 & 22,0 \\
\hline Ignorada & 18 & 4,0 & 18 & 5,0 & \multicolumn{2}{|c|}{0} \\
\hline Total & 512 & & 377 & & \multicolumn{2}{|c|}{135} \\
\hline
\end{tabular}

\section{Tabela 4}

Percentual dos óbitos perinatais (com idade gestacional conhecida) de acordo com o crescimento intra-uterino*, por faixa de idade gestacional. Maternidade Leila Diniz do Hospital Municipal Raphael de Paula Souza, Rio de Janeiro, 1999 a 2003.

\begin{tabular}{lcccc}
\hline $\begin{array}{l}\text { Idade gestacional } \\
\text { em semanas }\end{array}$ & AIG (\%) & PIG (\%) & GIG (\%) & Total \\
\hline$<28$ & 92,0 & 3,0 & 5,0 & 144 \\
28 a 31 & 78,0 & 19,0 & 3,0 & 103 \\
32 a 36 & 52,0 & 44,0 & 4,0 & 133 \\
$\geq 37$ & 55,0 & 32,0 & 12,0 & 114 \\
Total & 70,0 & 24,0 & 6,0 & 494 \\
\hline
\end{tabular}

* AIG=adequado para idade gestacional; PIG=pequeno para idade gestacional; GIG=grande para idade gestacional.
A mediana de idade gestacional (usando a variável idade gestacional estimada) para os óbitos perinatais foi 31 semanas. Os óbitos neonatais, comparados aos fetais, tiveram maior freqüência de idades gestacionais baixas. Não foi possível estimar a idade gestacional em $5 \%$ dos óbitos fetais e, portanto, em $4 \%$ dos óbitos perinatais (Tabela 3 ).

A relação da adequação do peso ao nascer à idade gestacional (em semanas) foi usada como proxy para crescimento intra-uterino restrito (CIUR). A freqüencia de PIG (pequenos para idade gestacional) foi menor nas idades gestacionais baixas $(<28$ semanas), tendendo a aumentar com o avanço da gestação e concentrando-se entre 32 e 36 semanas (Tabela 4).

Utilizando a Classificação de Wigglesworth, ${ }^{6}$ adaptada por Keeling, ${ }^{7}$ observou-se, para todas as faixas de peso, o predomínio de óbitos anteparto (grupo 1). Para os menores de $1500 \mathrm{~g}$, a prematuridade (grupo 3) foi a segunda categoria. Nas faixas de 1500 a $2499 \mathrm{~g}$, as causas específicas, que nesta população são representadas majoritariamente pelos casos de sífilis congênita, ocuparam a segunda posição. Já nos nascimentos com peso igual ou superior a $2500 \mathrm{~g}$, destacou-se também a asfixia intraparto com $17,8 \%$ dos casos. No total, malformação (grupo 2) foi a categoria menos freqüente. (Tabela $5)$.

Considerando os óbitos perinatais como um todo, foram encontradas como causas básicas mais freqüentes: afecções maternas (31\%), principalmente doenças hipertensivas e sífilis; complicações da placenta, membranas e cordão (24\%), com 
Tabela 5

Número absoluto e percentual de óbitos perinatais por faixa de peso, de acordo com a Classificação de Wigglesworth*, adaptada por Keeling et al.** Maternidade Leila Diniz do Hospital Municipal Raphael de Paula Souza, Rio de Janeiro, 1999 a 2003.

\begin{tabular}{|c|c|c|c|c|c|c|c|c|c|c|}
\hline \multirow[t]{2}{*}{ Peso ao nascer (g) } & \multicolumn{2}{|c|}{$\begin{array}{l}\text { Anteparto } \\
\text { (Grupo 1) }\end{array}$} & \multicolumn{2}{|c|}{$\begin{array}{c}\text { Malformação } \\
\text { (Grupo 2) }\end{array}$} & \multicolumn{2}{|c|}{$\begin{array}{c}\text { Prematuridade } \\
\text { (Grupo 3) }\end{array}$} & \multicolumn{2}{|c|}{$\begin{array}{c}\text { Asfixia intraparto } \\
\text { (Grupo 4) }\end{array}$} & \multicolumn{2}{|c|}{$\begin{array}{c}\text { Específicas } \\
\text { (Grupo 5) }\end{array}$} \\
\hline & $\mathrm{n}$ & $\%$ & $\mathrm{n}$ & $\%$ & $\mathrm{n}$ & $\%$ & $\mathrm{n}$ & $\%$ & $\mathrm{n}$ & $\%$ \\
\hline 500- 999 & 88 & 50,6 & 10 & 5,7 & 58 & 33,3 & 12 & 6,9 & 6 & 3,4 \\
\hline $1000-1499$ & 55 & 58,5 & 8 & 8,5 & 14 & 14,9 & 4 & 4,3 & 13 & 13,8 \\
\hline 1500-1999 & 40 & 57,0 & 8 & 11,5 & 4 & 5,7 & 3 & 4,3 & 15 & 21,5 \\
\hline $2000-2499$ & 32 & 57,0 & 3 & 5,5 & - & - & 6 & 10,7 & 15 & 26,8 \\
\hline$\geq 2500$ & 71 & 60,2 & 9 & 7,6 & - & - & 21 & 17,8 & 17 & 14,4 \\
\hline Total & 286 & 56,0 & 38 & 7,4 & 76 & 14,8 & 46 & 9,0 & 66 & 12,8 \\
\hline
\end{tabular}

Fonte: * Wigglesworth JS. Monitoring perinatal mortality. Lancet. 1980.6; * Keeling JW et al. Classification of perinatal death. Arch Dis Child. 1989.7

destaque para descolamento de placenta e amnionite; malformações congênitas ( $8 \%$ ), hipóxia intra-uterina intraparto $(6 \%)$; trabalho de parto prematuro $(6 \%)$; seguidos de infecções perinatais, crescimento intrauterino restrito e diabetes. Permaneceram com causa indeterminada $15 \%$ dos óbitos, considerando também os diagnósticos de hipoxia intra-uterina não especificada. As causas se distribuíram de forma diferenciada, ao analisarmos óbitos fetais e neonatais precoces, de acordo com a idade gestacional.

Os óbitos fetais com idade gestacional $<37$ semanas tiveram como causas mais freqüentes as afecções maternas (37\%), destacando-se novamente hipertensão e sífilis. Em seguida, as alterações placentárias, de membrana e cordão (26\%), e um elevado percentual de causas não definidas (21\%). Nos natimortos ocorridos a termo, a seqüência das causas foram: afecções maternas $(27 \%)$, alterações de placenta, membrana e cordão (25\%), causas não definidas $(17 \%)$ e asfixia perinatal $(12 \%)$.

Entre os óbitos neonatais precoces, no grupo de recém-nascidos pré-termo, foi possível explicar o mecanismo da prematuridade em $50 \%$ dos recémnascidos: afecções maternas (hipertensão, infecção urogenital, amniorrexe), complicações da placenta, membranas e cordão, malformações congênitas e infecções. As causas conseqüenciais mais comuns de óbito foram síndrome de angústia respiratória (doença de membrana hialina) e sepse neonatal.

Os recém-nascidos a termo tiveram como causa mais freqüente de óbito (33\%) asfixia ao nascer, acompanhada ou não de síndrome de aspiração meconial. A seguir vieram malformações congênitas $(23 \%)$ e infecções perinatais $(20 \%)$.

\section{Discussão}

Embora os dados deste trabalho se refiram a uma única maternidade, suas características de abrangência geográfica e cobertura do SUS permitem extrapolar os resultados para uma população de cerca de 300.000 mulheres em idade fértil dos bairros adstritos à maternidade. Comparando os resultados do presente trabalho com os de outros autores envolvendo partos hospitalares em outras cidades do Brasil,2,3,5 observam-se valores menores para asfixia intraparto (com exceção de Fortaleza) e prematuridade, enquanto os óbitos anteparto e aqueles por causas específicas foram bem mais elevados.

As características biológicas dos fetos e recémnascidos evidenciaram as questões do baixo peso, prematuridade e crescimento intra-uterino restrito, eventos já corroborados como associados à mortalidade perinatal. Quase $80 \%$ dos óbitos perinatais tinham menos de 37 semanas, sendo que os óbitos neonatais precoces apresentaram idades gestacionais mais baixas que os fetais. O crescimento intrauterino restrito só se tornou relevante após 28 semanas, concentrando-se na faixa de 32 a 36 semanas. O percentual de crescimento intra-uterino restrito encontrado para os óbitos fetais foi semelhante ao relatado por Conde-Agudelo et al., ${ }^{20}$ que pesquisaram tal percentual em países latino-americanos e utilizaram a curva de Williams. Pettersson et al. 21 encontraram $22 \%$ de insuficiência placentária associada ao crescimento intra-uterino restrito nos óbitos fetais em Estocolmo, analisando os anos de 1998 e 1999. Valor bem próximo - 23,4\% - foi o 
relatado por Borrell et al.,22 em Barcelona, no período de 1993 a 1997. Deve ser ressaltado que o crescimento intra-uterino restrito pode ser detectado no acompanhamento pré-natal e é passível de intervenção. 23

A interpretação dos dados relativos ao baixo peso na mortalidade perinatal deve ser feita separadamente para óbitos neonatais e fetais. No caso dos óbitos neonatais, nascer com baixo peso e/ou prematuridade desencadeia outros eventos - respiratórios, hemodinâmicos, metabólicos ou infecciosos, que dão seqüência à cadeia causal, até a ocorrência do óbito. No caso dos óbitos fetais, o nascimento em idade gestacional precoce e/ou com baixo peso é conseqüência de processos patológicos que culminaram com o decesso fetal, fechando a cadeia causal. 24

Cabe mencionar alguns aspectos metodológicos na análise das variáveis biológicas. A utilização dos dados antropométricos se mostrou bastante útil para estimar idade gestacional, quando não havia informação das outras fontes. O comprimento foi compatível com a idade gestacional estimada pela ultrasonografia em $65 \%$ dos casos neonatais e $60 \%$ dos fetais, quando esse dado era disponível. Por outro lado, confirmou-se a tendência de superestimação dos métodos clínicos neonatais. 25 Comparando a estimativa ultra-sonográfica e o método de Ballard, este mostrou superestimação maior ou igual a duas semanas em $65 \%$ dos casos. Em relação ao crescimento intra-uterino, a curva de Williams detectou $24 \%$ de fetos/neonatos com crescimento fetal restrito, sendo que após 31 semanas este valor sobe para $38 \%$. Caso fosse usada a curva de Lubchenco et al.,26 os valores seriam $15 \%$ e $23 \%$ respectivamente, mostrando a inadequação desta curva, que ainda é usada nos serviços de saúde e como instrumento de pesquisa.

Os coeficientes de mortalidade neonatal precoce foram comparáveis aos do município. Isto pode ser explicado, em parte, pela transferência de algumas gestantes de risco para unidades terciárias, mas sugere que, apesar dos indicadores socioeconômicos desfavoráveis da população usuária, a existência de suporte tecnológico e recursos humanos capacitados na atenção neonatal é capaz de minimizar as desigualdades. A Maternidade Leila Diniz fez parte de um projeto de qualificação do atendimento neonatal, no período de 1996-2001, com investimentos em recursos humanos e equipamentos. ${ }^{27} \mathrm{~A}$ análise dos coeficientes de mortalidade por faixa de peso corrobora a boa performance da unidade em relação à neomortalidade precoce. Embora pequeno, o contingente total de nascidos vivos de muito baixo peso, isto é, menores de $1500 \mathrm{~g}$ foi responsável por $60 \%$ dos óbitos neonatais e pequenos aumentos no número absoluto tiveram repercussão no coeficiente de mortalidade global. Para essa faixa de peso, considerando o período globalmente, o coeficiente de mortalidade neonatal foi $305 \%$ e o precoce foi $225 \%$, com pequena variação anual. Algumas maternidades consideradas terciárias da rede municipal do Rio de Janeiro apresentaram no ano de 2002 taxas de mortalidade neonatal precoce dos recém-nascidos de muito baixo peso ao nascer, variando entre $162 \%$ e $270 \% .28$ Estudos em outras localidades do Brasil mostram também taxas elevadas para essa faixa de peso: em Belo Horizonte, 5 no ano de 1999, a neomortalidade precoce foi $315 \%$; na região sul da cidade de São Paulo, 29 a neomortalidade total no período 2000-2001 foi 456\%.

Em contraponto, a mortalidade fetal se mantém mais elevada que a descrita para o Rio de Janeiro. Deve ser ressaltado que os óbitos fetais da AP4 se concentravam na Maternidade Leila Diniz, enquanto os neonatais precoces ocorrem em outras unidades públicas e algumas privadas, sugerindo diferenças socioeconômicas na gênese dos dois tipos de óbito.

Quando se aplicou a classificação de evitabilidade, o elevado percentual de óbitos anteparto aponta para problemas na atenção pré-natal, desde a completa ausência até a possibilidade de mau acompanhamento. Usando o peso de $1000 \mathrm{~g}$ como ponto de corte para viabilidade e excluindo as malformações congênitas letais, podemos estimar um número elevado de óbitos potencialmente evitáveis em torno de $60 \%$. Mesmo se usarmos um ponto de corte mais conservador - 1500 g, os óbitos evitáveis seriam cerca de $40 \%$ do total. A análise das causas básicas corrobora essa estimativa, já que afecções maternas passíveis de tratamento, como hipertensão e sífilis estão entre as causas mais freqüentes.

A comparação desta classificação com a de outras localidades deve ser criteriosa. Os demais estudos utilizaram uma base populacional praticamente completa. No caso da Maternidade Leila Diniz, cerca de $60 \%$ dos óbitos estão representados, principalmente os fetais. Isto explica o baixo percentual da categoria prematuridade, já que muitas gestantes de risco são transferidas para unidades terciárias. Por outro lado, o baixo percentual de asfixia perinatal fala a favor de bom cuidado obstétrico e de reanimação neonatal. O valor elevado da categoria de causas específicas se explica pelo esforço em investigar e monitorizar a sífilis congênita no Município do Rio de Janeiro, principalmente nas maternidades municipais, como a Maternidade onde se desenvolveu a investigação. 30 
As causas básicas revelam que algumas patologias agem no período perinatal de forma indistinta, resultando tanto em óbito fetal quanto neonatal. Um exemplo é a asfixia intraparto que contribuiu principalmente para os óbitos ocorridos em conceptos a termo. Outras patologias concentraram-se nos óbitos fetais, como as doenças hipertensivas e a sífilis, enquanto as malformações congênitas foram mais freqüentes nos neomortos. O elevado percentual de causa indeterminada tem duas explicações. A primeira, a forma de classificar o diagnóstico "anoxia intra-uterina", um dos mais freqüentes nos atestados de óbito fetal. Apesar de estar incluída no capítulo de transtornos respiratórios do período perinatal, 19 na maioria dos casos ocorridos na Maternidade Leila Diniz, essa terminologia foi mal empregada para classificar óbitos de causa não definida. Uma outra razão, em menor número de casos, foi a dificuldade em realizar necropsias na rede pública, impedindo a melhor apreensão da etiologia de alguns óbitos perinatais.

Os óbitos perinatais atendidos pela Maternidade Leila Diniz refletem aproximadamente a situação perinatal dos usuários do SUS em uma região do Rio de Janeiro e apontam para a necessidade de melhorar a oferta e qualificar a assistência pré-natal, além de continuar investindo na atenção neonatal de risco. Finalizando, deve ser enfatizada a investigação mais refinada da mortalidade. Devem ser valorizadas as descrições dos coeficientes por faixa de peso, a informação sobre idade gestacional e crescimento intra-uterino - de preferência usando a curva de Williams. Também deve ser aprimorada a determinação das causas do óbito, diminuindo o percentual de causas mal-definidas. Dessa forma, a investigação rotineira dos óbitos perinatais poderá ampliar o subsídio para as ações na área perinatal.

\section{Agradecimentos}

À Direção do Hospital Raphael de Paula Souza - Dr. Otávio Maia Porto e à Gerência da Maternidade Dra Ana Lúcia Alves, pelo apoio ao trabalho. À Comissão de Revisão de Óbitos da Maternidade Leila Diniz - Dr Luiz Otavio Mattos, Dra Nadir G. Santos e Dra Mônica Esposito, pelas valiosas sugestões na discussão dos casos; à Coordenação de Epidemiologia da Secretaria Municipal de Saúde, pela cessão do banco de dados do SINASC.

\section{References}

1. Fonseca SC, Coutinho ESF. Pesquisa sobre mortalidade perinatal no Brasil: revisão da metodologia e dos resultados. Cad Saúde Pública. 2004; 20 (Suppl 1): S7-S19.

2. Menezes AMB, Barros FC, Victora CG, Alves C, Rocha C, Albernaz E, Menezes FS, Jannke HA. Mortalidade perinatal em duas coortes de base populacional no Sul do Brasil: tendências e diferenciais. Cad Saúde Pública. 1996; 12 (Supl 1): 33-41.

3. Leite AJM, Marcopito LF, Diniz RLP, Silva AVS, Souza LCB, Borges JC, Sá HLC. Mortes perinatais no município de Fortaleza, Ceará: o quanto é possível evitar? J Pediatr. (Rio J) 1997; 73: 388-94.

4. Ribeiro VS, Silva AAM. Tendências da mortalidade neonatal em São Luís, Maranhão, Brasil, 1979 a 1996. Cad Saúde Pública. 2000; 16: 429-38.

5. Lansky S, França E, Leal MC. Mortes perinatais evitáveis em Belo Horizonte, Minas Gerais, 1999. Cad Saúde Pública. 2002; 18: 1389-400.

6. Wigglesworth JS. Monitoring perinatal mortality - A pathophysiological approach. Lancet. 1980; 2: 684-6.

7. Keeling JW, Macgillivray I, Golding J, Wigglesworth J, Berry J, Dunn PM. Classification of perinatal death. Arch Dis Child. 1989; 64: 1345-51.

8. Menezes AMB, Barros FC, Victora CG, Tomasi E, Halpern R, Oliveira ALB. Fatores de risco para mortalidade perinatal em Pelotas, RS, 1993. Rev Saúde Pública. 1998; 32: 209-16.
9. Lansky S, França E, Leal MC. Mortalidade perinatal e evitabilidade: revisão da literatura. Rev Saúde Pública. 2002; 36: 759-72.

10. Rio de Janeiro (Estado). Secretaria de Saúde. Coordenação de Programas de Epidemiologia. Gerência de Informações Epidemiológicas. Mortalidade perinatal no Rio de Janeiro. Disponível em: http://www.saude.rio.rj.gov.br /saude/ pubsms/media/Perinatal_7903.pdf. [2005 jan 23]

11. Gomes MASM, Campos TP, Giannini NM, Silva RI. Investigação dos óbitos fetais e neonatais nas maternidades municipais: Rio de Janeiro 1998. In: Resumos do IV Congresso Brasileiro de Epidemiologia; 1998 ago. 2-7; Rio de Janeiro. Rio de Janeiro: ABRASCO; 1998.

12. Hadlock FP, Harrist RB, Shah YP, King DE, Park SK, Sharman RS. Estimating fetal age using multiple parameters: a prospective evaluation in a racially mixed population. Am J Obstet Gynecol. 1987; 156: 955-7.

13. Usher R, McLean F. Intrauterine growth of live-born Caucasian infants at sea level: standards obtained from measurements in seven dimensions of infants born between 25 and 44 weeks of gestation. J Pediatr. 1969; 74: 901-10.

14. Miller HC, Hassanein K. Diagnosis of impaired fetal growth in newborn infants. Pediatrics. 1971; 48: 511-22.

15. Capurro H, Konichezky S, Fonseca D, Caldeyro-Barcia R. A simplified method for diagnosis of gestational age in the newborn infant. J Pediatr. 1978; 93: 120-2. 
16. Ballard JL, Khoury JC, Wedig K, Wang L, Eilers-Walsman BL, Lipp R. New Ballard score, expanded to include extremely premature infants. J Pediatr. 1991; 119: 417-23.

17. Williams RL, Creasy RK, Cunningham GC, Hawes WE, Norris FD, Tashiro M. Fetal growth and perinatal viability in California. Obstet Gynecol. 1982; 59: 624-32.

18. WHO (World Health Organization). Expert Committee on Physical Status. Physical status: the use and interpretation of anthropometry. Geneva; 1995. (Technical report series, 854).

19. OMS (Organização Mundial da Saúde). Classificação Estatística Internacional de Doenças e Problemas Relacionados à Saúde. 10. revisão. São Paulo: Centro Colaborador da OMS para a Classificação de Doenças em Português; 1995. v. 1.

20. Conde-Agudelo A, Belizan JM, Diaz-Rossello, JL. Epidemiology of fetal death in Latin America. Acta Obstet Gynecol Scand. 2000; 79: 371-8.

21. Pettersson K, Bremme K, Bottinga R, Hofsjö A, HulthénVarli I, Kublickas M, Norman M, Papadogiannakis N, Wånggren K, Wolff K. Diagnostic evaluation of intrauterine fetal deaths in Stockholm 1998-99. Acta Obstet Gynecol Scand. 2002; 81: 284-92.

22. Borrell C, Cirera E, Ricart M, Pasarin MI, Salvador J. Social inequalities in perinatal mortality in a Southern European city. Europ J Epidemiol. 2003; 18: 5-13.

23. Gardosi J. Customized growth curves. Clin Obstet Gynecol 1997; 40: 715-22.

24. Kiely JL. Some conceptual problems in multivariate analyses of perinatal mortality. Paediatr Perinat Epidemiol. 1991; 5: 243-57.

Recebido em 27 de junho de 2006

Versão final apresentada 11 de janeiro de 2008

Aprovado em 15 de fevereiro de 2008
25. Moraes CL, Reichenheim ME. Validade do exame clínico do recém-nascido para a estimação da idade gestacional: uma comparação do escore New Ballard com a data da última menstruação e ultra-sonografia. Cad Saúde Pública. 2000; 16: 83-94.

26. Lubchenco LO, Hansman C, Dressler M, Boyd E. Intrauterine growth as estimated from liveborn birth-weight data at 24 to 42 weeks of gestation. Pediatrics. 1963; 32: 793-800.

27. Gomes MASM. Assistência neonatal na Secretaria Municipal de Saúde do Rio de Janeiro: uma análise do período 1995-2000 [tese doutorado]. Rio de Janeiro: Instituto Fernandes Figueira da Fundação Oswaldo Cruz; 2002

28. Duarte JLMB, Mendonça GAS. Avaliação dos óbitos neonatais em recém-nascidos de muito baixo peso em quatro maternidades no Município do Rio de Janeiro, Brasil. Cad Saúde Pública 2005; 21: 387-95.

29. Almeida MF, França Junior I, Novaes HMD, Flores LPO, Siqueira AAF, Rodrigues LC, Montero CV. Mortalidade neonatal em $\mathrm{RN}<1500 \mathrm{~g}$ (RNMBPN) na Região Sul da Cidade de São Paulo (ZNMZP). In: Congresso Brasileiro de Epidemiologia, 2002, Curitiba, Paraná. Rev Bras Epidemiol. 2002; (Supl. Esp): 61. [resumo].

30. Saraceni V, Leal MC. Avaliação da efetividade das campanhas de eliminação da sífilis congênita na redução da morbidade e mortalidade perinatal: Rio de Janeiro. Cad Saúde Pública. 2003; 19: 1341-9. 